SHORT REPORT

\title{
Aquaporin-4 expression is increased in oedematous human brain tumours
}

\author{
S Saadoun, M C Papadopoulos, D C Davies, S Krishna, B A Bell
}

J Neurol Neurosurg Psychiatry 2002;72:262-265

Aquaporin-4 (AQP4) is a highly conserved water channel protein. In rats, AQP4 is expressed in astrocyte foot processes and is important in brain water homeostasis. AQP4 expression has not been investigated in non-neoplastic human brain or oedematous brain tumours, where water homeostasis is disrupted. Therefore, immunohistochemistry was used to study AQP4 expression in non-neoplastic and neoplastic human brain and blood-brain barrier permeability was assessed using contrast enhanced computed tomograms. AQP4 was present around microvessels in five specimens of non-neoplastic brain and five low grade (Daumas-Duport I or II) astrocytomas. AQP4 was massively upregulated in four and absent in one high grade (Daumas-Duport III or IV) astrocytoma. Massive upregulation of AQP4 was also found in reactive astrocytes in five metastatic adenocarcinomas. There was significant $(p<0.0001)$ correlation between blood-brain barrier opening and upregulated AQP4 expression. Increased AQP4 expression in high grade astrocytomas and adenocarcinomas may facilitate the flow of oedema fluid.

U ntil recently, water transport across cell membranes was thought to occur by simple diffusion. However, the rate of water transport across erythrocyte and kidney tubule cell membranes is higher than would be expected by simple diffusion alone. ${ }^{1}$ The reason for this was disclosed by the discovery of a family of water channel proteins, the aquaporins (AQPs), which facilitates the passage of water across cell membranes. ${ }^{1-2}$ To date, ten distinct AQPs have been characterised in mammals and over 30 other family members have been described in amphibians, insects, plants, and bacteria (Pubmed protein database).

In rats, $\mathrm{AQP} 4$ is expressed in perimicrovessel astrocyte foot processes $^{3}$ and alterations in AQP4 expression are associated with perturbations of brain water homeostasis. After brain injury $^{4}$ and focal ischaemia, ${ }^{5}$ there is upregulation of astrocyte AQP4 mRNA in regions where the blood-brain barrier is disrupted. After focal cerebral ischaemia, AQP4 knockout mice have less peri-infarct oedema than controls, ${ }^{6}$ suggesting that AQP4 aggravates oedema formation.

Little is known about AQP4 expression in normal human brain or brain tumours. Aggressive brain tumours cause peritumoural oedema, which increases patient morbidity and mortality. ${ }^{7-8}$ Oedema forms because leaky tumour microvessels allow fluid to enter the brain parenchyma from the microvessel lumen. ${ }^{8}$ The possibility that AQP4 expression is altered in oedematous brain to facilitate the formation or removal of oedema fluid was therefore investigated in astrocytomas and metastatic adenocarcinomas and compared with AQP4 expression in non-neoplastic brain. The pattern of AQP4 expression was correlated with blood-brain barrier permeability, assessed using contrast enhanced computed tomograms (CTs).

\section{MATERIALS AND METHODS \\ Patients}

The study was approved by St George's healthcare ethics committee. Fifteen patients (table 1) who underwent resection or biopsy of brain tumours at Atkinson Morley's Hospital in 1998-9 provided samples and were followed up for a year postoperatively. All specimens were examined by a neuropathologist without knowledge of the results described here. Astrocytomas were classified as low (low grade astrocytomas $=$ grades I-II) or high (high grade astrocytomas = grades III-IV) grade according to the DaumasDuport criteria." Each patient with a metastatic adenocarcinoma underwent excision of a solitary brain metastasis with an unknown primary. Non-neoplastic cerebral cortex was obtained from three patients who underwent temporal lobectomies for intractable epilepsy and from the entry sites of ventricular drains in two patients. All patients with brain tumours received dexamethasone ( $16 \mathrm{mg} /$ day, for $1-5$ days), compared with three of the five who contributed nonneoplastic brain tissue (table 1).

\section{Radiological imaging}

All patients underwent preoperative brain CT before and 30 minutes after administration of Omnipaque (Nycomed, Amersham, UK), injected as a $50 \mathrm{ml}$ intravenous bolus. Patients with low grade astrocytomas also had MRI, because most low grade astrocytomas are difficult to characterise on CT. Tumour size was quantified by measuring the maximum diameter of the enhanced region on CTs (for high grade astrocytomas and metastatic adenocarcinomas) or of the high signal region on T2 weighted MRIs (for low grade astrocytomas). All scans were reviewed by two neuroradiologists, without knowledge of the immunohistochemical results.

\section{Immunohistochemistry}

Tissue specimens were fixed in $10 \%$ formaldehyde $/ 0.9 \% \mathrm{NaCl}$ and processed into paraffin wax after 24 hours. Part of each specimen was processed for conventional histology to verify the nature of the tissue. Sections (10 $\mu \mathrm{m}$ thickness) were hydrated through graded alcohols and heated in sodium citrate buffer $\left(10 \mathrm{mM}, \mathrm{pH} 6,5 \mathrm{~min}, 95^{\circ} \mathrm{C}\right)$. They were incubated in $\mathrm{H}_{2} \mathrm{O}_{2}(0.3 \%, 10 \mathrm{~min})$ and blocked with normal goat serum (Vector, 1:60, $30 \mathrm{~min}$ ). The sections were then incubated with a polyclonal rabbit anti-rat AQP4 antibody (AB3068, Chemicon, $1: 100,3 \%$ goat serum, overnight, $4^{\circ} \mathrm{C}$ ). The immunoreacted sections were treated with a goat antirabbit biotinylated antibody (Sigma, 1:1000, 3\% goat serum, 1 hour) and incubated with avidin-biotin-horseradish peroxidase (Vector, $30 \mathrm{~min})$. Immunoreactivity was visualised by treating the specimens with $0.025 \%$ 3,3'-diaminobenzidine tetrachloride/

Abbreviations: AQP4, Aquaporin-4; AQPs, aquaporins; LGA, grades I-II-low grade astrocytoma, HGA, grades III-IV - high grade astrocytoma; GFAP, glial fibrillary acidic protein 
Table 1 Patient details, imaging, and histology data

\begin{tabular}{|c|c|c|c|c|c|c|c|c|c|c|c|}
\hline Histology & Grade & Age (y) & Sex & Side & Size $(\mathrm{cm})$ & Site & Dex & Adj & Surv & CT & $\begin{array}{l}\text { AQP4 } \\
\text { immunolabelling }\end{array}$ \\
\hline \multirow[t]{5}{*}{ Normal } & NA & 57 & $\mathrm{~F}$ & $R$ & & $\mathrm{Te}$ & $Y$ & $N$ & $Y$ & NE & 1 \\
\hline & NA & 65 & $M$ & $\mathrm{R}$ & & $\mathrm{Fr}$ & $N$ & $N$ & $Y$ & NE & 1 \\
\hline & NA & 42 & $\mathrm{~F}$ & $\mathrm{R}$ & & $\mathrm{Fr}$ & Y & $N$ & $Y$ & NE & 1 \\
\hline & NA & 36 & $M$ & L & & $\mathrm{Te}$ & Y & $\mathrm{N}$ & Y & $\mathrm{NE}$ & 1 \\
\hline & NA & 48 & $M$ & $\mathrm{R}$ & & $\mathrm{Te}$ & Y & $N$ & $Y$ & $\mathrm{NE}$ & 1 \\
\hline \multicolumn{2}{|c|}{ Mean (SD) or $\%$} & $50(12)$ & & & NA & & & & $100 \%$ & & \\
\hline \multirow[t]{5}{*}{ Astro } & $\|$ & 29 & $\mathrm{~F}$ & $\mathrm{R}$ & 6 & $\mathrm{Te}$ & Y & $\mathrm{N}$ & Y & $\mathrm{NE}$ & 1 \\
\hline & ॥ & 33 & $\mathrm{~F}$ & L & 2 & $\mathrm{Fr}$ & Y & $N$ & $Y$ & NE & 1 \\
\hline & II & 40 & $M$ & $\mathrm{R}$ & 5 & $\mathrm{~Pa}$ & Y & $N$ & Y & $\mathrm{NE}$ & 1 \\
\hline & $\|$ & 30 & $\mathrm{~F}$ & $\mathrm{R}$ & 5 & $\mathrm{Te}$ & Y & $\mathrm{N}$ & Y & $\mathrm{NE}$ & 1 \\
\hline & I & 30 & $M$ & $\mathrm{R}$ & 1 & $\mathrm{Te}$ & $Y$ & $N$ & Y & NE & 1 \\
\hline \multicolumn{2}{|c|}{ Mean (SD) or \% } & $32(5)$ & & & $3.8(2.2)$ & & & & $100 \%$ & & \\
\hline \multirow[t]{5}{*}{ Astro } & IV & 54 & $M$ & $R$ & 8 & $\mathrm{Te}$ & Y & $\mathrm{Ra}, \mathrm{Ch}$ & $\mathrm{N}$ & $E$ & 2 (0 in parts) \\
\hline & IV & 59 & $\mathrm{~F}$ & $\mathrm{R}$ & 5 & $\mathrm{Fr}$ & Y & $\mathrm{N}$ & $N$ & $\mathrm{E}$ & 2 \\
\hline & IV & 38 & $M$ & $\mathrm{R}$ & NK & $\mathrm{Te}$ & Y & $\mathrm{Ra}$ & Y & $\mathrm{E}$ & 2 \\
\hline & IV & 42 & $M$ & $\mathrm{R}$ & NK & $\mathrm{Te}$ & $Y$ & $\mathrm{Ch}$ & $Y$ & $\mathrm{E}$ & 2 (0 in parts) \\
\hline & IV & 48 & $M$ & $R$ & 4 & $\mathrm{~Pa}$ & $Y$ & $\mathrm{Ra}$ & $Y$ & $\mathrm{E}$ & 0 \\
\hline \multicolumn{2}{|c|}{ Mean (SD) or \% } & $48(9)$ & & & $5.7(2.1)$ & & & & $60 \%$ & & \\
\hline \multirow[t]{5}{*}{ Adeno } & NA & 61 & $\mathrm{~F}$ & C & 4 & $\mathrm{Ce}$ & Y & $\mathrm{Ra}$ & Y & $E$ & [2] \\
\hline & NA & 56 & $M$ & $\mathrm{R}$ & 2 & $\mathrm{Fr}$ & Y & $\mathrm{Ra}$ & $\mathrm{N}$ & $\mathrm{E}$ & {$[2]$} \\
\hline & NA & 67 & $\mathrm{~F}$ & $\mathrm{R}$ & 4 & $\mathrm{Te}$ & $Y$ & $\mathrm{Ra}$ & $Y$ & $\mathrm{E}$ & [2] \\
\hline & NA & 62 & $\mathrm{~F}$ & $R$ & 4 & $\mathrm{Fr}$ & $Y$ & $\mathrm{~N}$ & $\mathrm{~N}$ & $\mathrm{E}$ & [2] \\
\hline & NA & 76 & $\mathrm{~F}$ & L & 3 & $\mathrm{Ce}$ & Y & $N$ & $N$ & $\mathrm{E}$ & {$[2]$} \\
\hline \multicolumn{2}{|c|}{ Mean (SD) or \% } & $64(8)$ & & & $3.4(0.9)$ & & & & $40 \%$ & & \\
\hline
\end{tabular}

Adj, postoperative adjuvant treatment; Astro, astrocytoma; Adeno, adenocarcinoma; Ce, cerebellum; Ch, chemotherapy; CT, computed tomogram; Dex, dexamethasone; E, contrast enhancement; Fr, frontal; Grade, Daumas-Duport grade of astrocytoma; Histol, histology; L, left; N, no; NA, not applicable; $\mathrm{NE}$, no enhancement; NK, not known; Pa, parietal; R, right; Ra, radiotherapy; Surv, 1 year postoperative survival; Te, temporal; Y, yes; [ ], the cells are not tumour cells but reactive astrocytes.

$0.001 \% \mathrm{H}_{2} \mathrm{O}_{2}$ (Sigma). Sodium phosphate buffer ( $100 \mathrm{mM}, \mathrm{pH}$ 7.4) was used for washes, and antibody, serum, and diaminobenzidine dilutions. The tissue sections were counterstained with cresyl violet $(0.5 \%, 15 \mathrm{~min})$ and dehydrated through graded alcohols. They were then examined in a light microscope. Sections from all specimens were also immunoreacted with a polyclonal rabbit antihuman glial fibrillary acidic protein (GFAP) primary antibody (G9269, Sigma) to identify the cells expressing AQP4.

\section{Measurement of immmunolabelling}

Tissue sections were graded by two observers "blind" to the identity of each specimen according to the pattern and amount of AQP4 immunoreactivity as: $0=$ nil, $1=$ perimicrovessel (immunolabelling of astrocyte foot processes around microvessels only), 2 =widespread (immunolabelling of the cytoplasm of large numbers of cells, not necessarily associated with microvessels).

\section{Statistical analysis}

Fisher's exact test was used to compare proportions. One way ANOVA and the Student-Newman-Keuls test were used for multiple comparisons of parametric data.

\section{RESULTS}

AQP4 immunolabelling appeared as a brown deposit over cell processes surrounding microvessels in non-neoplastic brain (fig $\mathrm{l}$ A and B) and low grade astrocytoma (fig C). There was no difference in AQP4 immunolabelling between tissue sections from patients who received dexamethasone (fig l A) and those that did not (fig $1 \mathrm{~B}$ ). In four high grade astrocytomas there was strong immunolabelling over the cytoplasm of most tumour cells and/or reactive astrocytes that was not confined to the perimicrovessel region (fig $1 \mathrm{D}$ and E). Two high grade astrocytoma specimens had low grade areas in which AQP4 immunolabelling resembled that of non-neoplastic/LGA tissue (table 1). Two high grade astrocytoma specimens contained AQP4 immunonegative parts that were nonnecrotic and an entire non-necrotic high grade astrocytoma specimen was also AQP4 immunonegative (table 1). The AQP4 immunonegative cells in high grade astrocytomas appeared poorly differentiated and their nuclei stained strongly with cresyl violet. Large areas adjacent to metastatic adenocarcinomas exhibited increased AQP4 immunoreactivity, which was present over the cytoplasm of the immunolabelled cells (fig l F). AQP4 immunolabelled cells with scanty perinuclear cytoplasm and cytoplasmic processes were also scattered throughout the metastatic adenocarcinoma tissue itself (fig l G). AQP4 immunopositive cells in metastatic adenocarcinomas (fig $\mathrm{l} \mathrm{H}$ ) and astrocytomas (not shown) were GFAP immunopositive.

In all CT scans of patients with high grade astrocytoma and metastatic adenocarcinoma, the tumours enhanced after administration of intravenous contrast medium. No enhancement was seen on the scans of patients that provided non-neoplastic brain or low grade astrocytoma. There was a significant $(p<0.0001)$ correlation between the degree of contrast enhancement on CT and AQP4 immunolabelling of tissue from the same patient.

The mean age of patients with low grade astrocytoma was 16 years less than that of patients with high grade astrocytoma and 32 years less than that of patients with metastatic adenocarcinoma ( $p<0.05$ for both comparisons). There was no significant difference between the mean size of the different tumour types $(p=0.24)$. All of the patients with non-neoplastic brain tissue and low grade astrocytoma, $60 \%$ of those with high grade astrocytoma and $40 \%$ of those with metastatic adenocarcinoma survived 1 year postoperatively.

\section{DISCUSSION}

The current study demonstrates massive astrocyte AQP4 expression upregulation in oedematous brain tumours and surrounding tissue from patient populations that conform to published studies with regard to demographic 

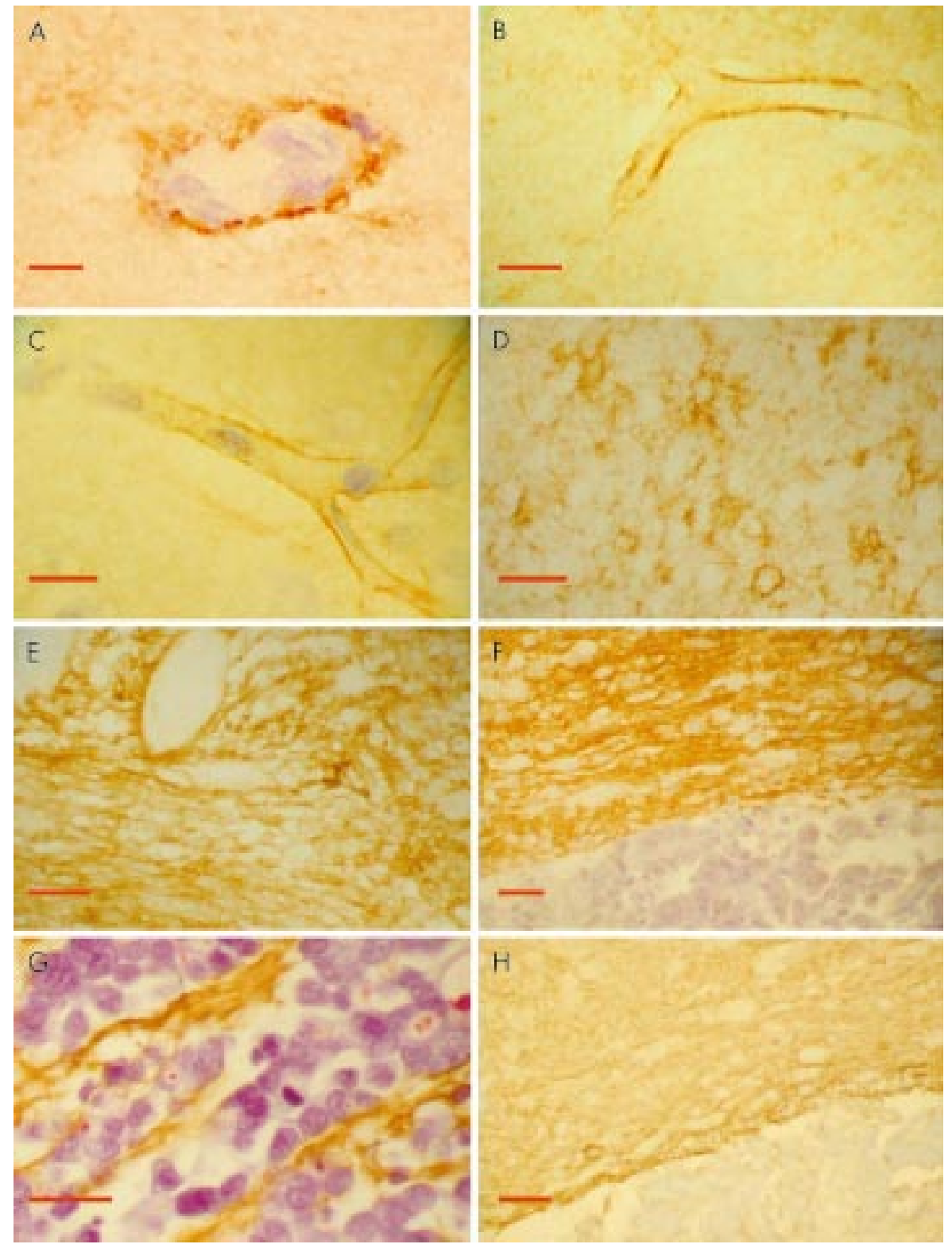

Figure 1 Light photomicrographs of human tissue immunolabelled for (A-G) AQP4 and (H) GFAP,

counterstained with cresyl violet. AQP4 is only expressed in cell processes around microvessels in $(A$ and $B$ ) normal human brain and (C) grade II astrocytoma. Patient (A) received dexamethasone and patient (B) did not. AQP4 immunolabels the cytoplasm of large numbers of cells in grade IV astrocytomas (D and E) and an adenocarcinoma metastasis (F). (G) Shows an adenocarcinoma in which AQP4 immunopositive cells with astrocyte morphology are "trapped"' among the tumour cells. (H) A similar section to (F) showing that the AQP4 immunopositive region also immunolabels for GFAP. Bar $=10$ $\mu \mathrm{m}$. characteristics. $^{9-11}$ AQP4 protein expression in non-neoplastic human brain was similar to that previously described for the rat. ${ }^{3}$ The AQP4 immunopositive cells are astrocytes because similar cells immunolabel for GFAP and have the morphology of astrocytes. In metastatic adenocarcinoma, these astrocytes are likely to be reactive but in high grade astrocytoma they include neoplastic astrocytes. The AQP4 immunonegative regions within some high grade astrocytoma specimens are composed of poorly differentiated tumour cells and may also lack reactive astrocytes (table 1). The blood-brain barrier is compromised in human astrocytomas and metastatic adenocarcinomas $^{12}$ and therefore, brain oedema in these patients is likely to be due to a balance between opening of the blood-brain barrier and AQP4 mediated water transport.

All non-neoplastic brain specimens investigated were histologically normal and their corresponding brain regions appeared normal on CT/MRI. Although three samples obtained from surgery for epilepsy (anterior temporal lobectomy for mesial temporal sclerosis) were used, these did not include the abnormal regions, but only parts of the lateral temporal lobes that were excised to gain access to the epileptic focus.
There was a significant correlation between increased AQP4 immunolabelling and the presence of contrast enhancement on CT. Such enhancement after administration of a bolus of intravenous iodinated contrast is a function of vascularity and the permeability of the blood-brain barrier. ${ }^{13-14}$ Peak blood concentrations are reached almost immediately followed by a rapid fall during the next 2 minutes as the contrast medium equilibrates between the plasma and the extracellular fluid of non-neural tissue. ${ }^{13}$ Subsequently, there is a more gradual fall in plasma concentration related to renal excretion. ${ }^{13}$ Thus, delayed CT enhancement, as assessed in the present study, reflects blood-brain barrier disruption rather than increased vascularity. ${ }^{13-14}$

In mouse cortical astrocytes, cultured in the presence of serum, AQP4 is expressed throughout the cytoplasm. ${ }^{15}$ This pattern of expression closely resembles that of tumour associated astrocytes in the present study, rather than that of non-neoplastic human (fig $1 \mathrm{~A}$ and B) or rodent ${ }^{3}{ }^{15}$ astrocytes. Therefore, AQP4 expression in normal astrocytes in vivo may be downregulated by factors released by other cells. In tumour associated astrocytes, AQP4 expression may be overexpressed due to the lack of inhibitory factors or in response to serum 
derived factors that reach the astrocytes through the open blood-brain barrier.

Dexamethasone, the drug widely used to treat brain tumour oedema, had no apparent effect on AQP4 expression in non-neoplastic human brain. As all patients with brain tumours received dexamethasone perioperatively, it is unclear whether dexamethasone had any effect on AQP4 expression in tumour patients.

The strong correlation between the presence of brain oedema and upregulated astrocyte AQP4 expression in human brain tumours (present study), hyponatraemic rats, ${ }^{16}$ rat brain injury $^{4}$ and rat focal cerebral ischaemia, ${ }^{5}$ suggests that increased AQP4 expression may be fundamental to the pathophysiology of brain oedema, regardless of aetiology. However, it remains unclear whether the AQP4 expressed in oedematous brain is functional or whether it enhances oedema fluid formation/clearance. The AQP4 knockout mouse ${ }^{17}$ may prove to be a useful tool to investigate these possibilities as would the development of specific AQP4 channel blockers and AQP4 may represent a novel pharmacological target for the design of anti-oedema agents.

\section{ACKNOWLEDGEMENTS}

We thank Dr P Wilkins, Mr R F Moss, and the neurosurgical nursing staff at Atkinson Morley's Hospital. This work was supported by a St George's Hospital Special Trustees grant to MCP.

\section{Authors' affiliations}

S Saadoun, D C Davies, Department of Anatomy and Developmental Biology, St George's Hospital Medical School, London SW17 ORE, UK S Krishna, Department of Infectious Diseases

M C Papadopoulos B A Bell, Department of Neurosurgery, Atkinson Morley's Hospital, London SW20 ONE, UK

Correspondence to: Dr D C Davies, Department of Anatomy and Developmental Biology, Cranmer Terrace, Tooting, St George's Hospital Medical School, London SW17 ORE, UK; daviesdc@sghms.ac.uk

Received 26 January 2001

In revised form 23 July 2001

Accepted 23 July 2001

\section{REFERENCES}

1 Verkman AS. Water transport across mammalian membranes. Am J Physiol 1996;48:C12-30.

2 King LS, Agre P. Pathophysiology of the aquaporin water channels. Ann Rev Physiol 1996:58:619-48.

3 Nielsen S, Nagelhus EA, Amiry-Moghaddam M, et al. Specialized membrane domains for water transport in glial cells: high-resolution immunogold cytochemistry of aquaporin-4 in rat brain. J Neurosci 1997; 17:171-80.

4 Vizuete ML, Venero JL, Vargas $C$, et al. Differential upregulation of aquaporin- 4 mRNA expression in reactive astrocytes after brain injury: potential role in brain oedema. Neurobiol Dis 1999;4:245-58.

5 Taniguchi $M$, Yamachita T, Kumura E, et al. Induction of aquaporin-4 water channel mRNA after focal cerebral ischemia in rat. Mol Brain Res 2000;78:131-7.

6 Manley GT, Fujimura M, Ma T, et al. Aquaporin-4 deletion in mice reduces brain edema after acute water intoxication and ischemic stroke. Nat Med 2000;6: 159-63.

7 Grieg NH. Brain tumors and the blood-brain barrier. In: Newelt EA, ed. Implications of the blood-brain barrier and its manipulation. Vol 2. New York: Plenum Press, 1989:77-106.

8 Papadopoulos MC, Saadoun S, Davies DC, et al. Emerging molecular mechanisms of brain tumour oedema. Br J Neurosurg 2001;15:101-8.

9 Daumas-Duport C, Scheithauer B, O'Fallon J, et al. Grading of astrocytomas: a simple and reproducible method. Cancer 1988;62:2152-65.

$10 \mathrm{Kim}$ TS, Halliday AL, Hedley-White AL, et al. Correlates of survival and the Daumas-Duport system for astrocytomas. J Neurosurg 1991;74:27-37.

11 Davis FG, Freels S, Grutsch J, et al. Survival rates in patients with primary malignant brain tumors stratified by patient age and tumor histological type and analysis based on surveillance, epidemiology and end results (SEER) data, 1973-91. J Neurosurg 1998;88:1-10.

12 Papadopoulos MC, Saadoun S, Woodrow CJ, et al. Occludin expression in microvessels of neoplastic and non-neoplastic human brain. Neuropathol Appl Neurobiol 2001,27:384-95.

13 Sage MR. Kinetics of water-soluble contrast media in the central nervous system. AJR Am J Roentgenol 1983;14:815-25.

14 Sage MR, Wilson AJ, Scroop R. Contrast media and the brain. The basis of CT and MR imaging enhancement. Neuroimaging Clin N Am 1998;8:695-707.

15 Nicchia GP, Frigeri A, Liuzzi GM, et al. Aquaporin-4-containing astrocytes sustain a temperature- and mercury- insensitive swelling in vitro. Glia 2000;31:29-38.

16 Vadia Z, Promeneur D, Doczi T, et al. Increased aquaporin-4 immunoreactivity in rat brain in response to systemic hyponatremia. Biochem Biophys Res Commun 2000;270:495-503.

17 Ma T, Yang B, Gillespie A, et al. Generation and phenotype of a transgenic knockout mouse lacking the mercurial-insensitive water channel aquaporin-4. J Clin Invest 1997;100:957-62. 